\title{
On the density of rational points on elliptic fibrations
}

\author{
F. A. Bogomolov \\ Courant Institute of Mathematical Sciences, N.Y.U. \\ 251 Mercer str. \\ New York, (NY) 10012, U.S.A. \\ e-mail: bogomolo@cims.nyu.edu \\ and \\ Yu. Tschinkel \\ Dept. of Mathematics, U.I.C. \\ 851 South Morgan str. \\ Chicago, (IL) 60607-7045, U.S.A. \\ e-mail: yuri@math.uic.edu
}




\section{Introduction}

Let $X$ be an algebraic variety defined over a number field $F$. We will say that rational points are potentially dense if there exists a finite extension $K / F$ such that the set of $K$-rational points $X(K)$ is Zariski dense in $X$. The main problem is to relate this property to geometric invariants of $X$. Hypothetically, on varieties of general type rational points are not potentially dense. In this paper we are interested in smooth projective varieties such that neither they nor their unramified coverings admit a dominant map onto varieties of general type. For these varieties it seems plausible to expect that rational points are potentially dense (see [2]).

Varieties which are not of general type can be thought of as triple fibrations $X \rightarrow Y \rightarrow Z$, where the generic fiber of $X \rightarrow Y$ is rationally connected, $Y \rightarrow Z$ is a Kodaira fibration with generic fiber of Kodaira dimension 0 and the base has Kodaira dimension $\leq 0$ (cf. [3]). In this paper we study mostly varieties of dimension 2 and 3 .

In dimension 2 the picture is as follows: Rationally connected surfaces are rational over some finite extension of $F$ and therefore the problem has an easy solution in this case. Rational points on abelian surfaces are potentially dense. In [2] density was proved for smooth quartic surfaces in $\mathbb{P}^{3}$ which contain a line and for elliptic fibrations over $\mathbb{P}^{1}$, provided they have irreducible fibers and a rational or elliptic multisection. We don't know the answer for general K3 surfaces. In this paper we prove that rational points are potentially dense on double covers of $\mathbb{P}^{2}$ ramified in a singular curve of degree 6.

In dimension 3 the situation is less satisfactory. For Fano 3-folds we have the following general

Theorem 1.1 Rational points are potentially dense on all Fano threefolds, with the possible exception of the family of double covers of $\mathbb{P}^{3}$ ramified in a smooth sextic surface.

This theorem follows from a case by case analysis of Fano threefolds, classified by Fano, Iskovskikh, Mori and Mukai (cf. [7], [8]). Most threefolds on the list are unirational and rational points on unirational varieties are potentially dense. The only exceptions for which unirationality is unknown 
for a general member of the corresponding family are: smooth quartics in $\mathbb{P}^{4}$ (treated in [2]), hypersurfaces of degree 6 in the weighted projective space $\mathbb{P}(1,1,1,2,3)$ (treated in this paper) and the double cover of a smooth sextic in $\mathbb{P}^{3}$.

At the moment, there are no general approaches to the problem of Zariskidensity of rational points, but a wealth of ad hoc methods appealing to specific geometric features for different classes of varieties. In particular, the situation is such that one can prove that rational points are potentially dense for certain varieties which are higher in the Kodaira hierarchy, but not for all varieties which are on the lower level. For example, density is well known for abelian varieties, whereas it is an open problem for general conic bundles over $\mathbb{P}^{2}$. It was pointed out to us by J.-L. Colliot-Thélène that Schinzel's hypothesis implies potential density for general conic bundles $X \rightarrow \mathbb{P}^{n}$ (with $X$ smooth) (cf. Theorem 4.2, p. 73 in [1]).

A natural strategy is to look at fibrations with fibers abelian varieties and to try to formulate sufficient conditions which would ensure that rational points are potentially dense. Our idea is roughly as follows: Let $\mathcal{A} \rightarrow B$ be a semiabelian variety over $B$ (defined over a number field $F$ ). Assume further that there exists a subvariety $\mathcal{M} \subset \mathcal{A}$ which is a multisection of the fibration and such that (potential) density holds for $\mathcal{M}$. Then there exists a finite extension of the groundfield $K / F$ such that $K$-rational points are dense in a Zariski dense set of fibers $\mathcal{A}_{b} \subset \mathcal{A}$ (for $b \in B$ ). The rest of the paper is devoted to the detailed study of conditions and situations when this naive idea actually works.

Acknowledgments. The second author is very grateful to Barry Mazur and Joe Harris for their interest, ideas and suggestions. We would like to thank J.L. Colliot-Thélène for his comments and the referee for the careful reading. The paper was written while both authors were enjoying the hospitality of the MaxPlanck-Institute in Bonn. The first author was partially supported by the NSF. The second author was partially supported by the NSA. 


\section{Elliptic fibrations}

Let $F$ be a field of characteristic zero and $B$ an irreducible $F$-scheme of dimension $\geq 1$. Let $j: \mathcal{E} \rightarrow B$ be a Jacobian elliptic fibration, i.e. an elliptic fibration with a zero section $e: B \rightarrow \mathcal{E}$, defined over $F$.

Definition 2.1 A saliently ramified multisection of $\mathcal{E}$ is an irreducible multisection $\mathcal{M}$ of $j$ which is defined over the algebraic closure $\bar{F}$ of $F$ and which is ramified in some non-singular fiber $\mathcal{E}_{b_{0}}=j^{-1}\left(b_{0}\right)\left(b_{0} \in B(\bar{F})\right)$.

Proposition 2.2 Let $j: \mathcal{E} \rightarrow B$ be a Jacobian elliptic fibration, defined over a number field $F$. If there exists a saliently ramified multisection $\mathcal{M}$ of $\mathcal{E}$ such that rational points on $\mathcal{M}$ are potentially dense then rational points on $\mathcal{E}$ are potentially dense.

Proof. Extending the groundfield $F$, if necessary, we can assume that $\mathcal{M}$ is defined over $F$ and that $F$-rational points are dense on $\mathcal{M}$. Clearly, $j(\mathcal{M}(F))$ is Zariski dense in $B$. We want to show that for almost all points $b \in$ $j(\mathcal{M}(F))$ (all outside a divisor in $B$ ) the points of intersection $\left(\mathcal{M} \cap \mathcal{E}_{b}\right)(F)$ generate an infinite subgroup of the group of rational points $\mathcal{E}_{b}(F)$.

Lemma 2.3 Let $j: \mathcal{E} \rightarrow B$ be a Jacobian elliptic fibration satisfying the conditions of Proposition 2.9. Then for any finite extension $K / F$ there exists a divisor $D \subset B$ (depending on $K$ ), such that for all $b \in j(\mathcal{M}(K)) \cap$ $(B \backslash D)(K)$ there exists a point $p_{b} \in \mathcal{M}(K) \cap \mathcal{E}_{b}(K)$ which is non-torsion in the group $\mathcal{E}_{b}(K)$.

Proof. Extending the groundfield we can assume that the ramification point $p_{0}$ of $\mathcal{M}$ in the smooth fiber $\mathcal{E}_{j\left(p_{0}\right)}$ is $F$-rational and that $\mathcal{M}$ is defined over $F$. For any $n$ the torsion points of order $n$ form a divisor $\Phi_{n}$ in $\mathcal{E}$. Thus, either our irreducible multisection $\mathcal{M}$ is contained in $\Phi_{n}$ for some $n$, or it intersects each $\Phi_{n}$ in a proper subvariety, which projects onto a proper subvariety of the base $B$. Assume that the multisection $\mathcal{M}$ is contained in $\Phi_{n}$ (for some $n$ ). One can argue as follows: fix an embedding of $\bar{F} \hookrightarrow \mathbb{C}$ and a neighborhood $U_{0} \subset B(\mathbb{C})$ containing $b_{0}$ such that for all $b \in U_{0}$ the fibers $\mathcal{E}_{b}(\mathbb{C})$ are smooth (this is possible by assumption). Then there exist a sequence of points $\left(b_{i}\right)_{i=1, \ldots, \infty}$ converging to $b_{0}$ and for each $b_{i}$ a pair of points $p_{i}, p_{i}^{\prime} \in\left(\mathcal{M} \cap \mathcal{E}_{b_{i}}\right)(\mathbb{C})$ (with $p_{i} \neq p_{i}^{\prime}$ ) such that both sequences $\left(p_{i}\right)_{i=1, . ., \infty}$ and $\left(p_{i}^{\prime}\right)_{i=1, . ., \infty}$ converge to $p_{0}$. For each $i$ the difference $\left(p_{i}-p_{i}^{\prime}\right) \in \mathcal{E}_{b_{i}}(\mathbb{C})$ is 
torsion of order $n$ and at the same time it converges to $0=e\left(b_{0}\right) \in \mathcal{E}_{b_{0}}(\mathbb{C})$. Contradiction.

Consider the second possibility: Merel's theorem about the boundedness of torsion for elliptic curves over number fields (cf. [6]) implies that there exists a number $n_{0}(F)$ such that the torsion subgroup of the group of rational points of every elliptic curve defined over $F$ is of order $\leq n_{0}(F)$. Let $D$ be the union of the images of $\Phi_{n} \cap \mathcal{M}$ (over all $n \leq n_{0}(F)$ ). This is a divisor in $B$. Then for all $b \in j(\mathcal{M}(F))$ which are not contained in $D$ there is an $F$-rational point $p_{b}$ in the fiber $\mathcal{E}_{b}$. It cannot be torsion of order $\leq n_{0}(F)$ on the one hand and there are no torsion points of order $>n_{0}(F)$. Therefore, it must be a point of infinite order.

An alternative argument would be to find a base change $B^{\prime} \rightarrow B$, étale at $j\left(p_{0}\right) \in B$ such that $\mathcal{M}$ pulls back to a section $\mathcal{M}^{\prime}$ of $\mathcal{E}^{\prime} \rightarrow B^{\prime}$. This section must be of infinite order in the Mordell-Weil group of $\mathcal{E}^{\prime}$. Then one can apply specialization (cf. [9]).

\section{Double covers of $\mathbb{P}^{2}$}

In this section we work over a number field, allowing, without further notice, finite extensions of the groundfield.

Let $R \subset \mathbb{P}^{2}$ be a reduced curve of degree 6 with a singular point $P$ and $\pi: D \rightarrow \mathbb{P}^{2}$ a double cover of $\mathbb{P}^{2}$ ramified in $R$. Consider the blow up of $\mathbb{P}^{2}$ at $P$ together with a natural linear system of lines in $\mathbb{P}^{2}$ passing through $P$. We obtain an elliptic fibration $\mathcal{E}_{P} \rightarrow \mathbb{P}^{1}$ on the desingularization $\mathcal{E}_{P} \rightarrow D$ in $\pi^{-1}(P)$. The fibration $\mathcal{E}_{P}$ has a natural section which is contained in the preimage of $P$ under this desingularization.

Proposition 3.1 Rational points on $D$ are potentially dense.

Proof. Surely, this is so if $\mathcal{E}_{P}$ is a rational variety or birational to a $\mathbb{P}^{1}$-bundle over an elliptic curve. This is the case if the curve $R$ contains multiple components or if the point $P$ has multiplicity $\geq 4$ on the curve $R \subset \mathbb{P}^{2}$. Indeed, if $R$ contains multiple components, then it is birational to a double cover of $\mathbb{P}^{2}$ ramified at a curve of degree at most 4 . Then it is either rational or birational to a $\mathbb{P}^{1}$-bundle over an elliptic curve. Similarly, if the 
point $P$ has multiplicity $\geq 4$ at $R$ then the corresponding bundle $\mathcal{E}_{P} \rightarrow \mathbb{P}^{1}$ is rational.

In general, $\mathcal{E}_{P} \rightarrow \mathbb{P}^{1}$ is a Jacobian elliptic fibration. We want to show the existence of a saliently ramified elliptic or rational multisection in order to use the Proposition 2.2. We have to consider several possibilities, depending on the curve $R$.

Definition 3.2 (Tangent correspondence) Let $R$ be a reduced curve in $\mathbb{P}^{2}$ and denote by $R^{0}$ the set of smooth points of $R$. The tangent correspondence $\mathrm{TC}(R) \subset R^{0} \times R^{0}$ is defined as follows: for any point $r \in R^{0}$ we consider the set of points $\mathrm{TI}_{r}(R)$ of transversal intersection of the tangent line $\mathbf{T}_{r}(R)$ through $r$ with $R$. Then

$$
\mathrm{TC}(R):=\left\{\left(r, \mathrm{TI}_{r}(R)\right)\right\} \subset R^{0} \times R^{0}
$$

Lemma 3.3 Assume that $\mathcal{E}_{P} \rightarrow \mathbb{P}^{1}$ is a Jacobian elliptic fibration and that there exists a tangent correspondence TC $(R)$ for the ramification curve $R$. Assume that $\mathrm{TC}(R)$ surjects onto some open part of a component of $R^{0}$ (under the second projection). Assume in addition that we can choose such a component to be different from a line through $P \in R$. Then $\mathcal{E}_{P} \rightarrow \mathbb{P}^{1}$ has a saliently ramified elliptic multisection.

Proof. The proof consists in a direct construction of two (different) elliptic curves contained in $D$ which are tangent to each other at a point which is smooth in both curves and in $D$. One of these curves is going to be a fiber of the Jacobian fibration $\mathcal{E}_{P} \rightarrow \mathbb{P}^{1}$ and the other a saliently ramified multisection of this fibration.

Denote by $L_{P, r} \subset \mathbb{P}^{2}$ the line joining $P$ and $r$ (for $r \neq P$ ). We can find a pair of smooth points $r, r^{\prime} \in R$ with $\left(r, r^{\prime}\right) \in \mathrm{TC}(R)$ with the property: The lines $\mathbf{T}_{r}(R)$ and $L_{P, r^{\prime}}$ both intersect in $r^{\prime}$ and are both transversal to the tangent line $\mathbf{T}_{r^{\prime}}(R)$ (by our assumptions on the tangent correspondence). We can also assume that the preimages of both lines are smooth elliptic curves on $D$ (since the points $r^{\prime}$ are allowed to vary in a smooth family which is not contained in a line through $P$ ). These elliptic curves are tangent in $D$ at the unique preimage $\pi^{-1}\left(r^{\prime}\right)$. The proof of this fact is local. Indeed, the image of the tangent space $\pi\left(\mathbf{T}_{\pi^{-1}\left(r^{\prime}\right)}(D)\right)$ coincides with the tangent line 
$\mathbf{T}_{r^{\prime}}(R)$. Since the lines $\mathbf{T}_{r}(R)$ and $L_{P, r^{\prime}}$ are transversal at $r^{\prime}$ both elliptic curves $\pi^{-1}\left(\mathbf{T}_{r}(R)\right)$ and $\pi^{-1}\left(L_{P, r^{\prime}}\right)$ are tangent at the point $\pi^{-1}\left(r^{\prime}\right)$ to the 1-dimensional subspace which is the kernel of the differential $\mathrm{d} \pi$ at $\pi^{-1}\left(r^{\prime}\right)$. Hence they are tangent at this point.

The assumptions of 3.3 are satisfied by most algebraic curves. More precisely

Lemma 3.4 Let $R \subset \mathbb{P}^{2}$ be any reduced curve with a point $P$ which contains either a component of degree $\geq 3$ or two conics or a conic and a line which doesn't contain P. Then the assumptions of 3.3 are satisfied.

Proof. Indeed, they are already satisfied for any irreducible curve of degree $\geq 3$. A general tangent line at a smooth point $r \in R$ intersects the curve transversally in at least one point $r^{\prime}$ and the point $r^{\prime}$ varies with $r$. Thus, if a curve $R$ contains such components, we are done. The case of two conics and a conic and a line not containing $P$ is evident.

Lemma 3.5 Let $L_{1}, \ldots, L_{6}$ be a configuration of 6 lines in $\mathbb{P}^{2}$ with no points of intersection of multiplicity $>3$. Then there are at least two points of intersection of multiplicity 2.

Proof. Exercise.

Corollary 3.6 Rational points are potentially dense on a double cover of any configuration $R$ of 6 lines in $\mathbb{P}^{2}$.

Proof. Consider two double points $P, Q$ and a line $L \in R$ which doesn't contain $P$ and $Q$. Then we have two Jacobian elliptic fibrations $\mathcal{E}_{P}$ and $\mathcal{E}_{Q}$ with fibers corresponding to the lines through $P$ and $Q$. Let $l \in L$ be a general point. Then the lines $L_{P, l}$ and $L_{Q, l}$ are transversal at $P$ to the line $L$. By the previous argument, the corresponding elliptic fibers of $\mathcal{E}_{P}$ and $\mathcal{E}_{Q}$ are tangent at the preimage of $P$ in $\mathrm{d} \pi$. Hence, both Jacobian elliptic fibrations have elliptic multisections tangent to smooth fibers and we can use our standard argument 2.2.

This concludes the proof of 3.1 . 


\section{Fano threefolds}

As described in the introduction, potential density of rational points on Fano threefolds is proved by combining classification and unirationality results. Smooth quartics for which unirationality is unknown were treated in [2].

Let $V_{1}$ be the Fano threefold given as a hypersurface of degree 6 in the weighted projective space $\mathbb{P}(1,1,1,2,3)$. It can be realized as a double cover $V_{1} \rightarrow V$ of the Veronese cone $V \subset \mathbb{P}^{6}$ ramified in an intersection of this cone with a smooth cubic hypersurface $C \subset \mathbb{P}^{6}$ which doesn't contain the vertex of $V$. We denote by $R$ the ramification divisor. If we blow up the singular point of the cone $V$ we obtain a smooth 3 -dimensional variety $V^{*} \simeq \mathbb{P}_{\mathbb{P}^{2}}(\mathcal{O}(2) \oplus \mathcal{O})$ which is birational to $V$. This projective bundle over $\mathbb{P}^{2}$ has two special sections corresponding to the decomposition into the direct sum above. We denote one of them by $\mathbb{P}_{0}:=\mathbb{P}_{\mathbb{P}^{2}}(\mathcal{O}(2))$ and the other by $\mathbb{P}_{\infty}:=\mathbb{P}_{\mathbb{P}^{2}}(\mathcal{O})$. The complement to the singular vertex of $V$ is isomorphic to the line bundle $\mathcal{O}(2)$ over $\mathbb{P}_{\infty}$. The double cover of $V^{*}$ ramified in $R$ (we will use the same notation $R$ for the preimage) will be denoted by $\pi: V_{1}^{*} \rightarrow V^{*}$. We denote by $p_{\infty}$ the projection $p_{\infty}: R \rightarrow \mathbb{P}_{\infty}$. On the reduced divisor $R$ we can define a subvariety $\operatorname{disc}(R)$ by the condition $\operatorname{rk}\left(\mathrm{d} p_{\infty}\right)<2$. This subvariety coincides with the subvariety of multiple intersection points of $R$ with the fibers of the $\mathbb{P}^{1}$ bundle $\mathbb{P}_{\mathbb{P}^{2}}(\mathcal{O}(2) \oplus \mathcal{O})$.

We observe that $V_{1}^{*}$ has a structure of a Jacobian elliptic fibration over $V_{1}^{*} \rightarrow \mathbb{P}_{\infty}^{2}$ where the elliptic fibers project to the lines of the cone $V^{*}$ and the zero section is $\mathbb{P}_{0}=\mathbb{P}_{\mathbb{P}^{2}}(\mathcal{O}(2))$. In this picture the preimage of $\operatorname{disc}(R)$ coincides with singular points of the fibers of the elliptic fibration $V_{1}^{*} \rightarrow \mathbb{P}_{\infty}^{2}$.

Theorem 4.1 Rational points on $V_{1}$ are potentially dense.

Proof. The Jacobian elliptic fibration $V_{1}^{*} \rightarrow \mathbb{P}_{\infty}^{2}$ has many double sections which are K3 surfaces. Any non-zero section $s$ of $\mathcal{O}(2)$ over $\mathbb{P}_{\infty}$ determines an embedding of $\mathcal{O} \hookrightarrow \mathcal{O} \oplus \mathcal{O}(2)$ and therefore a section $\mathbb{P}_{s}^{2} \simeq \mathbb{P}^{2} \hookrightarrow V^{*}$. The section $\mathbb{P}_{s}^{2}$ intersects the ramification divisor $R \subset V^{*}$ along a curve $R_{s} \subset \mathbb{P}_{s}^{2}$ of degree 6 . Thus we obtain a surface $D_{s} \subset V_{1}^{*}$ which is a double cover of $\mathbb{P}_{s}^{2}$ ramified in $R_{s}$. We will show that among the $D_{s}$ we can find one which is a saliently ramified multisection of the Jacobian elliptic fibration $V_{1}^{*} \rightarrow \mathbb{P}_{\infty}^{2}$ and on which rational points are potentially dense. Then we apply 2.2 . 
We choose a smooth point $P$ on the reduced ramification divisor $R$ such that the differential of the projection to the base $\mathbb{P}^{2}$ of $\mathbb{P}_{\mathbb{P}^{2}}(\mathcal{O} \oplus \mathcal{O}(2))$ is an isomorphism. This is always possible unless $R$ is a $\mathbb{P}^{1}$-fibration over a curve of degree 6 in $\mathbb{P}^{2}$. But in this case the initial cubic hypersurface $C \subset \mathbb{P}^{6}$ must contain the vertex of the Veronese cone $V$. It is worth noticing that the corresponding smooth threefold is a $\mathbb{P}^{1}$-fibration over a $\mathrm{K} 3$ surface and therefore not rationally connected. By the same reasoning, $\operatorname{disc}(R)$ is a curve in $R$.

Continuing the proof, we can now guarantee the existence of a family of sections $\mathcal{F}_{P} \simeq H^{0}\left(\mathbb{P}^{1}, \mathcal{O}(2)\right)$ such that for all $s \in \mathcal{F}_{P}$ the section $\mathbb{P}_{s}^{2}$ is tangent to the ramification divisor $R \subset V^{*}$ at $P$. This is possible because we can find a quadratic function on $\bar{F}^{2}$ with given value and given first differential at $P$, which defines the desired section $s$ of $\mathcal{O}(2)$ on $\mathbb{P}_{\infty}$. Moreover, locally around $P$ the second differential of the function describing $R$ with respect to $\mathbb{P}_{s}^{2}$ can be chosen at will. Then the curve $R_{s}$ is a singular curve of degree 6 with singular point $P$. This implies that the corresponding double cover $D_{s}$ is a singular surface with an isolated singular point at the preimage of $P$. Moreover, notice that $P \notin \operatorname{disc}(R)$. Therefore, in every curve $R_{s}$ there exists an open neighborhood $U_{s}(P) \subset R_{s}$ of $P$ which doesn't intersect the curve $\operatorname{disc}(R) \subset R$. This shows that every such $D_{s}$ is a saliently ramified multisection of the fibration $V_{1}^{*} \rightarrow \mathbb{P}_{\infty}^{2}$.

Now we want to use this multisection which is tangent to points contained in smooth fibers of the Jacobian elliptic fibration $V_{1}^{*} \rightarrow \mathbb{P}_{\infty}^{2}$ along $U_{s}(P)$ in our argument 2.2. We need to show that rational points are potentially dense on $\mathcal{E}_{P, s}$ (or, equivalently on $D_{s}$ ). But this is the content of Proposition 3.1 . Therefore, rational points are potentially dense on $V_{1}$.

Remark 4.2 In fact, we have a choice of $D_{s}$. In particular, in most situations we can choose $D_{s}$ such that the ramification curve $R_{s}$ has two singular points of multipliticity two. Then we obtain two Jacobian elliptic fibrations with some non-singular fibers of one fibration tangent to non-singular fibers of the other.

\section{References}


[1] J.-L. Colliot-Thélène and P. Swinnerton-Dyer, Hasse principle and weak approximation for pencils of Severi-Brauer and similar varieties, J. Reine Angew. Math., 453:49-112, (1994).

[2] J. Harris and Yu. Tschinkel, Rational points on quartics, Preprint 1998.

[3] J. Kollár, Y. Miyaoka and Sh. Mori, Rational curves on Fano varieties, In Classification of irregular varieties (Trento, 1990), volume 1515 of Lecture Notes in Math., pages 100-105. Springer, Berlin, 1992.

[4] Yu. I. Manin, Notes on the arithmetic of Fano threefolds, Compositio Math., 85(1):37-55, 1993.

[5] B. Mazur, The topology of rational points, Experiment. Math., 1(1):3545, 1992.

[6] L. Merel, Bornes pour la torsion des courbes elliptiques sur les corps de nombres, Invent. Math., 124(1-3):437-449, 1996.

[7] Sh. Mori and Sh. Mukai, Classification of Fano 3-folds with $B_{2} \geq 2$, Manuscripta Math., 36(2):147-162, 1981/82.

[8] Sh. Mori and Sh. Mukai, On Fano 3-folds with $B_{2} \geq 2$, In Algebraic varieties and analytic varieties (Tokyo, 1981), volume 1 of Adv. Stud. Pure Math., pages 101-129. North-Holland, Amsterdam, 1983.

[9] J. Silverman, Heights and the specialization map for families of abelian varieties, J. Reine Angew. Math., 342:197-211, 1983. 